\title{
A Multistudy Cross-Sectional and Experimental Examination Into the Interactive Effects of Moral Identity and Moral Disengagement on Doping
}

\author{
Nicholas Stanger and Susan H. Backhouse \\ Leeds Beckett University
}

\begin{abstract}
Moral identity and moral disengagement have been linked with doping likelihood. However, experiments testing the temporal direction of these relationships are absent. The authors conducted one cross-sectional and two experimental studies investigating the conjunctive effects of moral identity and moral disengagement on doping likelihood (or intention). Dispositional moral identity was inversely (marginally), and doping moral disengagement, positively, associated with doping intention (Study 1). Manipulating situations to amplify opportunities for moral disengagement increased doping likelihood via anticipated guilt (Study 2). Moreover, dispositional moral identity (Study 2) and inducing moral identity (Study 3) were linked with lower doping likelihood and attenuated the relationship between doping moral disengagement and doping likelihood. However, the suppressing effect of moral identity on doping likelihood was overridden when opportunities for moral disengagement were amplified. These findings support multifaceted antidoping efforts, which include simultaneously enhancing athlete moral identity and personal responsibility alongside reducing social opportunities for moral disengagement.
\end{abstract}

Keywords: experiment, guilt, moderation, moral judgment, sport psychology

Sport is a social context where people can behave in ways that have positive implications for others, such as congratulating teammates and opponents for good play. However, at the same time, the competitive and social aspect of sport can also provide opportunities and temptations for players to commit antisocial behaviors, which are typically defined as behaviors intended to harm or disadvantage others, such as cheating (Sage, Kavussanu, \& Duda, 2006). Due to the potential focus on outperforming others in sport, some people may be more inclined to commit morally questionable behaviors than in everyday contexts (e.g., Bredemeier \& Shields, 1986; Kavussanu, Boardley, Sagar, \& Ring, 2013). Accordingly, considering its inherent social and competitive nature, sport provides a relevant context to understand moral action.

One topical and prevalent issue surrounding sport that can be considered a moral issue is doping. Doping can reflect an antisocial act, particularly if undertaken intentionally, as it can provide an unfair and illegitimate advantage over others (Kaye \& Boardley, 2012). Under the World Anti-Doping Code (World Anti-Doping Agency, 2015), it is each athlete's personal responsibility to ensure that no prohibited substance enters his or her body. Failure to do so violates the spirit of sport, presents potential

(C) 2020 The Authors. Published by Human Kinetics, Inc. This is an Open Access article distributed under the terms of the Creative Commons Attribution-NonCommercial-NoDerivatives 4.0 International License, CC BY-NC-ND 4.0, which permits the copy and redistribution in any medium or format, provided it is not used for commercial purposes, no modifications are made, appropriate credit is given, and a link to the license is provided. See http://creativecommons.org/licenses/ by-nc-nd/4.0. This license does not cover any third-party material that may appear with permission in the article. For commercial use, permission should be requested from Human Kinetics, Inc., through the Copyright Clearance Center (http://www. copyright.com)

The authors are with the Carnegie School of Sport, Leeds Beckett University, Leeds, United Kingdom. Stanger (N.Stanger@leedsbeckett.ac.uk) is corresponding author. harm to the athlete, and can also impede the rights of others (Koller, 2008; Pope et al., 2013). Committing an anti-doping rule violation, particularly the intentional use of a prohibited substance to enhance performance, can be considered a form of cheating that casts a shadow over sport and fundamentally challenges the integrity of those involved (e.g., Koller, 2008). Therefore, research investigating factors underpinning such behaviors, from a moral standpoint, helps develop and strengthen anti-doping intervention models. Currently, an experimentally driven evidence base does not exist, and present-day interventions and psychological deterrence measures are largely modeled on assumptions from crosssectional research. To this end, this research aims to address this gap in the evidence base.

\section{Social Cognitive Theory of Moral Thought and Action}

The social cognitive theory of moral thought and action (Bandura, 1991) provides a framework that can contribute to our understanding of prohibited substance use in sport. Central to this theory is that self-censure in the form of emotions is essential in the regulation of immoral behaviors that go against one's moral standards. For instance, athletes may refrain from taking prohibited substances because of the unpleasant emotional consequences they anticipate experiencing if they were to take such substances (e.g., guilt). Accordingly, some qualitative evidence has revealed that guilt is a deterrent of doping (Kirby, Moran, \& Guerin, 2011), and anticipated guilt has been negatively associated with doping in crosssectional studies (e.g., Boardley, Smith, Mills, Grix, \& Wynne, 2017; Ring \& Kavussanu, 2018). Furthermore, self-censure has been proposed to be a stronger deterrent of committing immoral actions than fear of social sanctions (e.g., punishments, bans), because many acts can go undetected by others (Bandura, 1991). 
Therefore, research investigating the regulatory role of emotion and the factors that influence these mechanisms in prohibited substance use can strengthen the quality and effectiveness of anti-doping programs.

Bandura (1991) suggested people can commit antisocial actions without experiencing the usual unpleasant emotional consequences via the use of moral disengagement. Specifically, moral disengagement refers to a set of psychosocial mechanisms or processes that people use to justify committing transgressive behaviors by cognitively distorting the act or its consequences, reducing personal responsibility for the behavior, or by blaming or dehumanizing the victim. Therefore, moral disengagement can allow athletes to commit an anti-doping rule violation by rationalizing the act as more acceptable (or less wrong) and reduce or eliminate the typical anticipated guilt that would usually regulate doping.

Although Bandura (1991) mainly discusses moral disengagement as a process, research investigating moral disengagement typically measures it as an individual disposition reflecting people's propensity to use mechanisms of moral disengagement (e.g., Moore, 2015). A range of cross-sectional studies have found that dispositional moral disengagement in sport is positively associated with susceptibility to doping (Hodge, Hargreaves, Gerrard, \& Lonsdale, 2013), and that dispositional moral disengagement toward doping is positively associated with doping intention (Lucidi et al., 2008), doping likelihood (Ring \& Kavussanu, 2018), and reported doping (Boardley et al., 2017; Lucidi et al., 2008). Recent cross-sectional studies have also revealed that the positive relationships between moral disengagement and doping likelihood (Ring \& Kavussanu, 2018), as well as reported doping (Boardley et al., 2017), are mediated via reduced anticipated guilt.

Qualitative research has revealed that people (e.g., bodybuilders) justify use of image and performance-enhancing drugs (IPEDs) via six of the eight mechanisms of moral disengagement (Boardley \& Grix, 2014; Boardley, Grix, \& Dewar, 2014; see Boardley \& Kavussanu, 2011), namely, displacement of responsibility (e.g., a coach putting pressure on their athletes may deflect athletes taking personal responsibility to take IPEDs to improve performance), diffusion of responsibility (e.g., athletes perceiving that everyone else is taking IPED's), distortion of consequences (e.g., minimizing the harm caused by taking a banned substance), advantageous comparison (making IPED use appear less harmful by comparing it with more harmful acts), euphemistic labeling (e.g., using terms such as "juice" to make IPED use sound less immoral), and moral justification (e.g., taking IPED for helping other athletes). Yet, the research designs employed so far investigating the link between moral disengagement and doping have not yet been able to determine the temporal direction of this relationship or the situational characteristics that may induce moral disengagement. Specifically, it is not clear in cross-sectional and qualitative research whether moral disengagement leads to doping or whether doping leads to athletes justifying their behavior via moral disengagement. Thus, experimental evidence is needed to also examine moral disengagement as a process to determine whether moral disengagement is amenable and can affect the regulatory role of emotions, moral judgments, and athletes' doping likelihood. In other words, experimental evidence can examine whether we can reduce moral disengagement and whether this would lead to a reduction in doping. In a recent metaanalysis (Ntoumanis, Ng, Barkoukis, \& Backhouse, 2014), it was emphasized that there is a profound lack of experimental evidence into the antecedents of doping, highlighting the strengths of experimental-based research and the need to move beyond cross-sectional designs to inform anti-doping programs.

\section{Moral Identity}

Moral identity is another key factor in the regulation of moral conduct (Narvaez \& Lapsley, 2009). Specifically, moral identity refers to a cognitive schema that people hold about their moral character and reflects the importance that one places on being a moral person, such as being compassionate, kind, and fair (Aquino $\&$ Reed, 2002). Previous research has revealed that moral identity is positively associated with negative emotional reactions to unethical behavior (Stets \& Carter, 2011) and negatively linked with antisocial sport behavior (Kavussanu, Stanger, \& Ring, 2015). Recently, researchers have also demonstrated that dispositional moral identity is inversely associated with doping likelihood (Kavussanu \& Ring, 2017). These findings provide preliminary support that moral identity could be an important factor in reducing prohibited substance use. However, from a social cognitive perspective (e.g., Aquino, Freeman, Reed, Lim, \& Felps, 2009), the strength of the relationship between moral identity and doping likelihood may be influenced by the situational characteristics. For instance, in situations that enhance the accessibility of one's moral identity, this can enhance the motivation and likelihood to behave morally. However, in situations that reduce accessibility to one's moral identity (e.g., via situations that drive self-focused interests), this could increase the likelihood of engaging in antisocial conduct. Indeed, moral identity has been significantly and negatively linked with doping likelihood in situations where doping was perceived to have a cost to the athlete (e.g., risk of negative side effects to health; financial costs), but moral identity was not significantly associated with doping likelihood in situations where doping may have a benefit for the athlete (e.g., recovery from injury, high rewards, encouraged by coach; Ring, Kavussanu, Lucidi, \& Hurst, 2019). Given these findings, it is possible that the strength of the relationship between moral identity and doping likelihood could be influenced by situational factors that could include the extent to which players may have opportunities to apply mechanisms of moral disengagement.

Although researchers have shown that moral identity and doping likelihood are negatively related indirectly via moral disengagement (e.g., Kavussanu \& Ring, 2017), moral identity may also potentially play a moderating role on the relationship between dispositional moral disengagement and doping. For instance, Aquino, Reed, Thau, and Freeman (2007) examined American students' moral judgments about punitive retaliatory responses to the perpetrators of the 9/11 terrorist attacks, and found that students who reported higher advantageous comparison (a mechanism of moral disengagement) were associated with perceiving more severe punitive acts in retaliation toward the perpetrators of these terrorist attacks (e.g., using any means to kill those responsible for these acts, compared with capturing and trialing those responsible and imprisoning them for life if found guilty) as less morally wrong. However, an interactive effect was noted between dispositional moral identity and advantageous comparison on moral judgment, whereby the relationship between advantageous comparison and perceptions that severe retaliation was less morally wrong was stronger in individuals reporting low moral identity, with this relationship being negated in people who reported high moral identity. Although this study was in the context of moral judgments toward a different behavior, it is possible that moral identity may moderate the relationship between dispositional moral disengagement and doping. Specifically, when moral identity is high, this may reduce athletes' likelihood of doping even if they are inclined to morally disengage. However, research has yet to address this possibility. 
Researchers have also highlighted that moral identity can be considered a dispositional characteristic as well as a construct that can also be activated via priming moral traits, such as honesty, kindness, and fairness, and thereby making the moral aspect of peoples' identity more accessible in memory (e.g., Aquino et al., 2007, 2009). Previous research has found that priming moral identity reduced athletes' likelihood of engaging in antisocial behaviors in sport (e.g., Kavussanu et al., 2015). In addition, Aquino et al. (2007) found an interactive effect between priming moral identity and dispositional moral disengagement (i.e., advantageous comparison) on negative emotional reactions toward prisoner of war abuse. Specifically, advantageous comparison was associated with less negative emotional reactions to prisoner of war abuse, but this relationship was attenuated in individuals whose moral identity was primed. However, no research has manipulated moral identity to see if it influences doping likelihood. Research investigating the role of dispositional moral identity, and the effect of manipulating the salience of moral identity, on doping could provide evidence for the tailoring of anti-doping interventions and prevention strategies that target moral identity for enhancing the salience of moral values to reduce doping likelihood.

\section{The Present Research}

A key limitation of anti-doping research to date is the lack of experimental evidence to inform interventions that aim to prevent doping in sport (e.g., Ntoumanis et al., 2014). This research aims to experimentally investigate the effects of manipulating frequently used mechanisms of moral disengagement on the regulatory role of anticipated guilt and athletes' likelihood to dope. Thus, this research will extend the current evidence base through looking at moral disengagement both as a dispositional variable and a situational process. Moreover, moral identity may undermine the effect of moral disengagement may play in rationalizing prohibited substance use. Therefore, this research will aim to determine whether dispositional moral identity, and inducing moral identity, may be associated with lower doping likelihood even if athletes are susceptible to morally disengage.

In Study 1, we aimed to initially establish whether dispositional moral identity and doping moral disengagement were linked with athletes' doping intentions. We also aimed to investigate whether moral identity moderated the link between doping moral disengagement and doping intention. Specifically, we examined whether moral disengagement was more strongly (and positively) related with doping intention for athletes with low moral identity than those with high moral identity.

In Study 2, we aimed to test whether experimentally manipulating situational moral disengagement facilitated doping likelihood, reduced anticipated guilt, and perceived such behaviors as less morally wrong and more acceptable. It was also expected that inducing situational moral disengagement will increase athletes' likelihood to dope via reducing anticipated guilt. We also examined whether dispositional moral identity was negatively linked with doping likelihood as well as whether moral identity moderated the relationship between dispositional doping moral disengagement and athletes' doping likelihood in situations where they have low (low moral disengagement condition) and high (high moral disengagement condition) opportunities for moral disengagement.

To extend on Study 2, in Study 3 we primed moral identity to test if this influenced athletes' anticipated guilt, moral judgments, and doping likelihood across situations where athletes have low or high opportunities to morally disengage. It was expected that the effects of priming moral identity will replicate those found for dispositional moral identity in Study 2. We also aimed to test whether priming moral identity strengthened any inverse relationship between dispositional moral identity and doping likelihood, and negated any positive link between doping moral disengagement and doping likelihood.

\section{Study 1}

\section{Methods}

Participants. Participants were 239 team sport players (160 men and 79 women), with an average age of $19.40(S D=1.95)$ years. They competed in soccer $(n=82)$, rugby $(n=53)$, field hockey $(n=$ $44)$, netball $(n=17)$, cricket $(n=17)$, basketball $(n=12)$, volleyball $(n=6)$, lacrosse $(n=4)$, and American football $(n=4)$. Participants competed in their respective sports at international/national $(22 \%)$, regional/county (60\%), and club (18\%) level for an average of 9.35 $(S D=4.01)$ years.

Measures. Doping moral disengagement: Dispositional doping moral disengagement was measured using the 6-item scale developed by Lucidi et al. (2008) to assess moral disengagement toward doping. Participants were asked to rate their level of agreement to statements on a 7-point Likert type scale, anchored by 1 (strongly disagree) to 7 (strongly agree). An example item is "It is not right to condemn those who use banned substances to improve their performance because many do the same." Each item assesses one of the six mechanisms of moral disengagement used to justify doping in previous research. Psychometric support for the scale has been provided with an alpha coefficient of .84 (Lucidi et al., 2008). Similarly, in this study, the scale comprised of a satisfactory internal consistency $(\alpha=.80)$. We also checked the factor structure of the scale using confirmatory factor analysis which revealed an excellent fit to the data, Satorra-Bentler $\chi^{2}(9)=12.73, p=.18$; root mean square error of approximation $=.04$, comparative-fit index $=.99$, Tucker-Lewis index $=.98$, standardized root mean square residual $=.03$, with factor loadings ranging between .42 and .90; thus, supporting the unidimensional structure of the scale. A mean of the items was used in analyses.

Moral identity: Moral identity was measured using the 5-item internationalization subscale from the moral identity scale (Aquino $\&$ Reed, 2002). This scale assesses the extent to which a range of moral traits is central to the individual's self-concept (Aquino \& Reed, 2002). Specifically, participants were presented with nine moral traits (e.g., caring, compassionate, fair, and honest) and asked to think about a person who possesses these traits. Then, participants were asked to answer five statements in relation to these traits (e.g., I strongly desire to have these characteristics) on a 7-point scale anchored from 1 (strongly disagree) to 7 (strongly agree). Aquino and Reed (2002) have provided psychometric support for the scale including factorial, convergent, discriminant, and predictive validity as well as internal consistency $(\alpha=.83)$. A satisfactory internal consistency $(\alpha=.79)$ was also found in this sample. A mean was calculated and used in analyses.

Doping intention: A single item was used to assess athletes' current doping intention in relation to taking a banned substance in the next 3 months. Specifically, participants were asked to what extent they intended to use a banned substance to improve their sport performance or physical appearance at least once during the next 3 months on a 6-point Likert scale with anchors of 1 (definitely no) to 6 (definitely yes), similar to previous research (e.g., Lucidi et al., 2008). 
Social desirability: Social desirability was measured using the 13-item short-form Marlowe-Crowne Social Desirability Scale (Reynolds, 1982). Participants were asked a series of questions (e.g., "I'm always willing to admit it when I make a mistake") and asked to answer true or false to each item. For analysis, a sum score was calculated whereby true was coded as 1 , and false coded as 0 , but scores were reversed for items where false was the socially desirable response.

Procedure. Following ethical approval from the researchers' university ethics committee (Leeds Beckett University), participants were approached by one of the researchers or a research assistant. After reading the participant information sheet and completing a consent form, participants provided demographic information and completed the measures described above. ${ }^{1}$ Participants were thanked and offered a hot drink voucher for their participation.

\section{Results}

Preliminary Analysis. Data screening revealed 10 pieces of missing data $(0.17 \%)$. Due to the small frequency of missing items, and all participants responded to the single item for doping intention, we used the mean of the remaining items from the respective subscale for the other variables (Tabachnick \& Fidell, 2013). Screening for the normality of data via skewness and kurtosis values revealed no significant deviation from normality for moral identity and moral disengagement (skewness and kurtosis between -2 and 2; West, Finch \& Curran, 1995). However, doping intention was heavily skewed and comprised multiple outliers (skewness = 4.48; kurtosis $=21.88$ ) and thereby demonstrated significant deviation from normality. This potentially may be due to the nature of the variable whereby some athletes may not be intending to take banned substances in the next 3 months, or they may not be open to admit intentions to take banned substances (i.e., only 25 of the 243 athletes reported some doping intention; i.e., $>1$ on the scale), which is an issue evident in previous research (Barkoukis, Lazuras, Tsorbatzoudis, \& Rodafinos, 2013; Lucidi et al., 2008). Therefore, we use Spearman's rank for correlations that included this variable (Tabachnick \& Fidell, 2013).

Correlational Analysis. Descriptive statistics and correlations are presented in Table 1. Correlational analyses revealed that moral disengagement was positively associated with doping intention and inversely related with social desirability and gender (i.e., men reported higher moral disengagement than women), whereas moral identity was negatively associated (marginally) with doping intention, and positively linked with social desirability and gender (i.e., women reported higher moral identity than men). Moral identity and moral disengagement were inversely related, and doping intention was not linked with social desirability.

Regression Analyses. To examine whether dispositional moral identity moderated the relationship between moral disengagement and doping intention, we conducted moderated hierarchical regression analyses (i.e., Aiken \& West, 1991) on doping intention whereby the variables were entered into regression models in a three-step process. We entered gender and social desirability (as covariates) in Step 1. Dispositional moral identity and doping moral disengagement were entered in Step 2, and the product term (interaction) of mean-centered moral identity and meancentered doping moral disengagement were entered in Step 3 (Aiken \& West, 1991). Due to the issues with normality for the outcome variable in the model (i.e., doping intention), we used bootstrapping with 1,000 bootstrap samples to adjust, and provide more robust, parameter estimates in the model.

As presented in Table 2, gender and social desirability contributed $2 \%$ of the variance in doping intention whereby men reported higher doping intentions than women. Dispositional moral identity

\section{Table 1 Descriptive Statistics and Correlations With Doping Intention in Study 1} $(N=239)$

\begin{tabular}{lcccccc}
\hline & $\mathbf{M}$ & $\mathbf{S D}$ & $\mathbf{1}$ & $\mathbf{2}$ & $\mathbf{3}$ & $\mathbf{4}$ \\
\hline 1. Doping moral disengagement & 2.12 & 1.04 & & & & \\
2. Moral identity & 5.86 & 0.90 & $-.19^{* *}$ & & & \\
3. Doping intention & 1.18 & 0.67 & $.29^{* * *}$ & $-.13^{\#}$ & & \\
4. Social desirability & 6.63 & 2.43 & $-.17^{*}$ & $.14^{*}$ & -.06 & \\
5. Gender & - & - & $-.13^{*}$ & $.25^{* * *}$ & $-.15^{*}$ & .12 \\
\hline
\end{tabular}

Note. Doping moral disengagement and moral identity were measured on a 1-7 scale. Doping intention was measured on a 1-6 scale. Pearson's correlations were used for the links between moral identity, moral disengagement, social desirability, and gender, whereas Spearman's correlations were used for links with doping intentions, which are presented in italics. ${ }^{\#} p<.06 . * p \leq .05 . * * p<.01 . * * * p<.001$

Table 2 Hierarchical Regression Analysis for Moral Identity and Dispositional Doping Moral Disengagement on Doping Intention in Study $1(N=239)$

\begin{tabular}{|c|c|c|c|c|c|c|}
\hline Step & Predictor variable & $B$ & SE B & Confidence interval & $\Delta R^{2}$ & $\Delta \boldsymbol{F}$ \\
\hline \multirow[t]{2}{*}{1} & Gender & $-0.18^{*}$ & 0.08 & {$[-0.33,-0.04]$} & .02 & 1.96 \\
\hline & Social desirability & 0.00 & 0.01 & {$[-0.03,0.03]$} & & \\
\hline \multirow[t]{2}{*}{2} & Moral identity & -0.07 & 0.06 & {$[-0.22,0.05]$} & .19 & $28.35 * * *$ \\
\hline & Moral disengagement & $0.27 * *$ & 0.08 & {$[0.12,0.42]$} & & \\
\hline 3 & Moral identity $\times$ Moral disengagement & -0.12 & 0.10 & {$[-0.25,0.11]$} & .04 & $13.26 * * *$ \\
\hline
\end{tabular}

Note. Bootstrap coefficients are reported.

$* p<.05 . * * p<.01 . * * * p<.001$. 
and doping moral disengagement contributed an additional 19\% variance in doping intentions whereby doping moral disengagement was a significant positive predictor of doping intention, but dispositional moral identity was not a significant predictor. In Step 3, although a Moral identity $\times$ Moral disengagement interaction added a significant unique variance to the model (4\% additional variance), the bootstrap coefficients for the interaction were not significant. Therefore, suggesting no significant moderating effect of moral identity on the relationship between doping moral disengagement and doping intention.

\section{Discussion}

The findings from Study 1 provide further insight into the relationships between moral identity, doping moral disengagement, and doping intention. As expected moral identity was negatively (marginally), and moral disengagement was positively, associated with doping intention in the correlational analyses. These findings are aligned to previous studies showing a negative relationship between moral identity and doping likelihood (e.g., Kavussanu \& Ring, 2017), and a positive link between moral disengagement and doping likelihood (e.g., Kavussanu \& Ring, 2017; Ring \& Kavussanu, 2018), and doping susceptibility (e.g., Hodge et al., 2013). However, it should be noted the link between moral identity and doping intention only reflected a small effect size in this study, which was notably weaker than that found between moral identity and doping likelihood in previous research which was more reflective of a small to medium effect size (e.g., $r=-.27$; Kavussanu \& Ring, 2017).

We also tested whether moral identity moderated the relationship between moral disengagement and doping intention. However, following bootstrapping, there was no support for this moderating effect. This lack of moderating effect and the relatively weak relationship between moral identity and doping intention may be due to several reasons. ${ }^{2}$ First, only around $10 \%$ (25 out of 243 athletes) indicated any intention to engage in doping in the next 3 months. This lack of variance for doping intention is common in previous research (e.g., Lucidi et al., 2008; Whitaker, Long, Petroczi, \& Backhouse, 2013) as participants may not be particularly open to express such intentions, and/or they may not hold such intentions. Accordingly, a combination of the doping intention measurement alongside the requirement to have a much larger sample size that comprised a sufficient number of participants who reported any intention to dope may have accounted for the potential lack of significant interaction in this cross-sectional study. Therefore, researchers have measured doping likelihood using hypothetical scenarios (e.g., Barkoukis, Lazuras, Lucidi, \& Tsorbatzoudis, 2015; Huybers \& Masanov, 2012; Kavussanu \& Ring, 2017) to assess doping in a more indirect way, as people tend to be more open to report potential likelihood of doping. Also, the context and situation that participants compete in sport are likely to differ across participants which may influence their intentions to engage in doping. To help control for situational factors, in the next study we used scenarios to manipulate opportunities for moral disengagement to determine whether this influences athletes' doping likelihood, and to examine whether dispositional moral identity may be linked with doping likelihood across situations differing in opportunities for moral disengagement. We also examined whether dispositional moral identity may moderate the link between dispositional doping moral disengagement and doping likelihood across situations differing in opportunities for moral disengagement.

\section{Study 2}

\section{Methods}

Participants. Participants were 72 team sport players (46 men and 26 women) with an average age of $19.21(S D=1.22)$ years. They competed in a range of team sports with the majority participating in soccer $(n=24)$, rugby $(n=13)$, field hockey $(n=$ $7)$, or netball $(n=8)$. Participants competed in their respective sports at international/national $(11 \%)$, regional/county $(58 \%)$, or club $(31 \%)$ levels for an average of $8.53(S D=4.46)$ years.

Moral Disengagement Manipulation. We developed scenarios to manipulate three mechanisms of moral disengagement that have been reported as being most frequently used to justify performanceenhancing drug use in previous research (e.g., Boardley \& Grix, 2014; Boardley et al., 2014). Specifically, similar to previous research (Stanger, Kavussanu, Boardley, \& Ring, 2013), we developed scenarios to manipulate displacement of responsibility (supportive coach vs. pressurizing coach), diffusion of responsibility (not suspecting other athletes are doping vs. suspecting other athletes are doping) and advantageous comparison (not suspecting other athletes are committing other rule-breaking behaviors vs. perceiving other athletes are committing other rule-breaking behaviors) whereby the scenarios were adapted to induce low moral disengagement or high moral disengagement. For instance, to manipulate displacement of responsibility in the low moral disengagement condition participants were asked to read the following situation:

Imagine that recently your sport performances have not been as good as last season. Your coach has been very supportive and loyal, instilling you with confidence and encouragement that you will soon re-discover your form. You have noticed that one of your teammates' performances have really improved recently. After practice one day, your teammate tells you that he/she has been using a banned performance-enhancing substance and there are no tests to detect it.

In the high moral disengagement condition, participants read the same situation but the part in italics was changed to "Your coach is putting intense pressure on you to perform better and threatens that he/she may drop you from the squad if you do not find a way to improve your performances soon." The full manipulations for moral disengagement mechanisms are presented in the Appendix. Participants read all three scenarios for low moral disengagement in the same block, and the three scenarios for high moral disengagement in the same block, respectively. The ordering of whether participants read the low moral disengagement or high moral disengagement scenarios was fully counterbalanced. Participants were provided with a 3-min break between the low and high moral disengagement conditions. To further reduce the potential of order effects, the ordering of the scenarios within conditions was also counterbalanced across participants. Thus, there were six combinations of scenarios for the low moral disengagement scenarios multiplied by the six combinations for the high moral disengagement scenarios, resulting in 36 different possible combinations. Accordingly, we ended with a sample size of 72 (multiple of 36). ${ }^{3}$

Dispositional Measures. Dispositional doping moral disengagement, moral identity, and social desirability: Doping moral disengagement $(\alpha=.72)$, moral identity $(\alpha=.83)$, and social desirability were measured as per Study 1. Both dispositional moral disengagement and moral identity were measured at least 3 days, but no longer than 30 days, prior to this experimental session. This was done 
to help reduce any potential priming effect from completing these questionnaires immediately prior to completing the measures described below in the experimental session.

Experimental Session Measures. Anticipated guilt: After reading each scenario, participants were asked to rate their anticipated guilt if they were to take the banned substance in the scenario on a 5-point scale anchored by 1 (not at all) to 5 (extremely) using the 5-item guilt scale from the State Shame and Guilt Scale (Marschall, Saftner, \& Tangney, 1994). An example item is "remorse, regret." Marschall et al. (1994) provided psychometric support for the guilt scale (e.g., $\alpha=.82$ ), and this approach has been adopted to assess anticipated guilt in previous research (e.g., Kavussanu et al., 2015; Stanger et al., 2013). We also included two items from the pride subscale from the State Shame and Guilt Scale to act as filler items, so that positively valenced items were included to reduce potentially leading responses with having only "negatively framed" items throughout. As the mean score for anticipated guilt was highly correlated for the three scenarios within the low moral disengagement ( $r s=.76$ to .84 ) and for the three scenarios within the high moral disengagement ( $r \mathrm{~s}=.79$ to .80 ) condition, we calculated a mean score across the three scenarios within each condition for analyses.

Moral judgment: After reading each scenario, participants were asked to rate their judgments in relation to the scenario using two questions. Specifically, participants were asked to indicate the extent they perceived the behavior in the scenario as acceptable on a 7-point scale anchored from 1 (not at all acceptable) to 7 (very acceptable), and similar to previous research (e.g., Kavussanu et al., 2015) the extent they perceived the behavior in the scenario to be morally wrong on a 7-point scale from 1 (not at all wrong) to 7 (very wrong). As responses about whether the behavior in the scenarios were perceived as acceptable or wrong were highly correlated across the scenarios within the low moral disengagement (acceptable: $r s=.73$ to .79 ; morally wrong: $r \mathrm{~s}=.48$ to .68 ) and within the high moral disengagement (acceptable: $r \mathrm{~s}=.71$ to .82 ; wrong: $r \mathrm{~s}=.63$ to .72 ) condition, we calculated an overall mean score for acceptable and wrong for both low and high moral disengagement conditions.

Doping likelihood: After reading each scenario, participants were asked to indicate the likelihood they were to take the banned substance in each situation on a 7-point scale anchored from 1 (not at all likely) to 7 (very likely). This approach has been adopted to measure likely behavior in a range of previous studies (e.g., Kavussanu et al., 2015; Kavussanu \& Ring, 2017; Stanger et al., 2013). As the mean score for doping likelihood across the scenarios was highly correlated within the low moral disengagement $(r s=.60$ to .63$)$ and high moral disengagement $(r s=.71$ to .81) conditions, we calculated a mean score for doping likelihood across the three scenarios within each condition.

Manipulation check: To check the effectiveness of the moral disengagement manipulation, we included three questions adapted from the moral disengagement in sport scale to make them relevant to doping (Boardley \& Kavussanu, 2007); specifically, an item for each of the three mechanisms being manipulated. Participants were asked to imagine themselves taking the banned substance in the previous three scenarios (i.e., after each block of three scenarios for high and low moral disengagement) and asked to indicate their level of agreement for each item on a 7-point scale, anchored from 1 (strongly disagree) to 7 (strongly agree). Specifically, participants were asked: "I should not be blamed for using banned substances because everyone seems to be using them" (diffusion of responsibility), "I should not take responsibility for using the banned substance because my coach reinforces such behavior" (displacement of responsibility), and "compared to some of the things my teammates and opponents do, taking a banned substance is not that bad" (advantageous comparison). A mean score was calculated for the three items for both the low and high moral disengagement condition.

Procedure. Following ethical approval from the researchers' university ethics committee (Leeds Beckett University), participants were approached by one of the researchers or a research assistant. After reading the participant information sheet and completing a consent form, participants provided demographic information and completed the measures for doping moral disengagement, moral identity, and social desirability. At least 3 days (but no more than 30 days) later, participants attended an experimental session. At the start of this session, participants were provided the information sheet and a second consent form to confirm that they still agreed to participate and understood what participation would involve. Participants then completed a second demographics sheet so responses could be matched with the previous measures to help maintain anonymity. ${ }^{4}$ During this session, participants were informed that they will read two blocks of three scenarios and would be asked to answer a range of questions after reading each scenario. Participants were asked to read each scenario carefully until the situation was clear in their mind. After reading each scenario, participants completed the measures described above to assess their moral judgment, anticipated guilt, and doping likelihood in relation to that situation. Participants read and completed measures for all three scenarios for the low moral disengagement in the same block, and the three scenarios for the high moral disengagement condition in the same block. At the end of each block, participants completed the manipulation check items. After reading and completing the items for each of the six scenarios, participants were debriefed, thanked for the participation, and offered a $£ 5$ multistore voucher for taking part.

\section{Results}

Preliminary Analyses. Data screening revealed only three cases of missing data $(0.05 \%)$, so we used the mean of the remaining items from the respective subscale when this occurred (Tabachnick \& Fidell, 2013), and screening for skewness and kurtosis values revealed no significant deviation from normality.

Manipulation Check. Prior to the main analyses, we first checked whether the manipulation was effective by conducting a repeated-measure analysis of covariance (ANCOVA) on the manipulation check scores for the low and high moral disengagement condition (controlling for gender and social desirability). The results yielded significant differences between the two conditions on the manipulation check, $F(1,69)=18.68, p<.001$, $\eta_{\mathrm{p}}^{2}=.21$, reflective of a medium to large effect size. Specifically, participants reported higher moral disengagement in the high moral disengagement condition $(M=2.35, S D=1.39)$ compared with the low moral disengagement condition $(M=1.97, S D=$ $1.25)$; thus, supporting the effectiveness of our manipulation of moral disengagement.

The Effect of Moral Disengagement on Judgment, Guilt, and Doping Likelihood. To check for the effect of moral disengagement on moral judgment, anticipated guilt, and doping likelihood, we conducted a one-way repeated-measures multivariate ANCOVA controlling for gender and social desirability. A significant multivariate effect for moral disengagement was found, $F(4,66)=6.96, p<.001, \eta_{\mathrm{p}}^{2}=.30$. As presented in Table 3, 
follow-up repeated-measure ANCOVAs revealed significant differences for each variable. Participants perceived taking the banned substance as more acceptable and less morally wrong, anticipated feeling less guilt if they were to take a banned substance, as well as reported greater likelihood to dope in the high moral disengagement condition compared with the low moral disengagement condition.

To test whether the effect of moral disengagement on doping likelihood was mediated via anticipated guilt, we conducted within-subject mediation analysis using bootstrapping (e.g., Hayes, 2009) via the MEMORE macro for regression analyses (Montoya \& Hayes, 2017) in Statistical Package for the Social Sciences (IBM Corp., Armonk, NY). The model was run with 5,000 bootstrap samples to estimate the indirect effect; when the confidence intervals of the indirect effect do not cross zero then there is evidence of mediation. As depicted in Figure 1, moral disengagement positively predicted doping likelihood and negatively predicted anticipated guilt, and anticipated guilt negatively predicted doping likelihood. When controlling for anticipated guilt, the effect of moral disengagement on doping likelihood was attenuated. Mediation analyses revealed that the indirect effect of moral disengagement on doping likelihood via anticipated guilt was significant (indirect effect $=$ $0.162 ; 95 \%$ confidence interval $[0.03,0.33])$. Therefore, suggesting moral disengagement increased doping likelihood partially via reducing anticipated guilt.

The Moderating Effect of Dispositional Moral Identity. To address our next study purpose regarding whether dispositional moral identity moderated the relationship between doping moral disengagement and doping likelihood under situations where players have differing levels of opportunities to morally disengage, we conducted moderated hierarchical regression analyses (i.e., Aiken \& West, 1991) on doping likelihood in the low moral disengagement and high moral disengagement conditions separately. The variables were entered into regression models as per Study 1 (see Table 4).
Under conditions of low moral disengagement, dispositional moral identity was a negative predictor, and doping moral disengagement was a positive predictor, of doping likelihood. A significant Moral identity $\times$ Moral disengagement interaction was also revealed. Specifically, simple slope analyses revealed that moral disengagement was a significant positive predictor of doping likelihood $(b=0.70, \beta=0.78, t=4.91, p<.001)$ in individuals with low moral identity, whereas in individuals with high moral identity, moral disengagement was not a significant predictor $(b=$ $0.17, \beta=0.19, t=1.21, p=.23$; see Figure 2). In contrast, under conditions of high moral disengagement, dispositional moral disengagement remained a significant positive predictor, whereas moral identity was no longer a negative predictor of doping likelihood. Moreover, no Moral identity $\times$ Doping moral disengagement interaction was found.

\section{Discussion}

Our findings support theory (e.g., Bandura, 1991) by highlighting that under conditions where athletes are more likely to morally disengage, players' judge doping as more acceptable and less wrong, their anticipated guilt reduces, and doping likelihood increases. Moreover, our findings indicate that moral disengagement increased athletes' doping likelihood in part by reducing the regulatory role of anticipated guilt. Our findings thereby support theoretical predictions (e.g., Bandura, 1991), and are aligned to previous research highlighting that moral disengagement is a positive predictor of athletes' doping likelihood (e.g., Lucidi et al., 2008; Ring \& Kavussanu, 2018) via reducing anticipated guilt (e.g., Ring \& Kavussanu, 2018).

Moral identity was negatively associated with doping likelihood when opportunities for moral disengagement were low, but this relationship was overridden when opportunities for moral disengagement were amplified. Moreover, this study also provided novel findings in relation to the moderating role of moral identity on the relationship between dispositional doping moral disengagement and doping likelihood. Specifically, moral identity moderated

Table 3 Effect of Moral Disengagement on Doping Likelihood, Anticipated Guilt, and Moral Judgment in Study 2

\begin{tabular}{lccccccc}
\hline & \multicolumn{2}{c}{$\begin{array}{c}\text { Low moral } \\
\text { disengagement }\end{array}$} & & \multicolumn{2}{c}{$\begin{array}{c}\text { High moral } \\
\text { disengagement }\end{array}$} \\
\cline { 2 - 7 } & $\boldsymbol{M}$ & $\mathbf{S D}$ & & $\boldsymbol{M}$ & $\mathbf{S D}$ & $\mathbf{F ( 1 , 6 9 )}$ & $\boldsymbol{\eta}_{\mathbf{p}}^{2}$ \\
\hline Morally wrong & 6.41 & 0.92 & 6.02 & 1.20 & $7.65^{* *}$ & .10 \\
Acceptable & 1.59 & 0.94 & 2.13 & 1.32 & $9.25^{* *}$ & .11 \\
Anticipated guilt & 3.95 & 0.95 & 3.66 & 1.08 & $22.88^{* * *}$ & .25 \\
Likelihood to dope & 1.59 & 0.84 & 2.26 & 1.46 & $14.41^{* * *}$ & .17 \\
\hline
\end{tabular}

Note. Gender and social desirability were included as covariates.

$* * p<.01 . * * * p<.001$

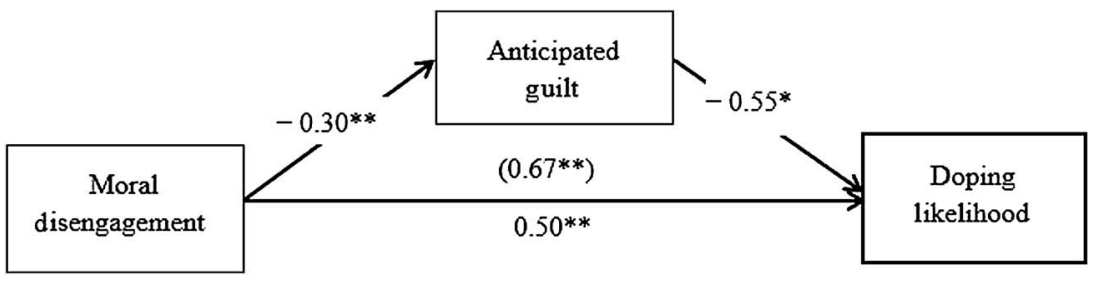

Figure 1 - Model for the mediating role of anticipated guilt in Study 2. Unstandardized regression coefficients are presented. The uncorrected coefficient for the effect of moral disengagement on doping likelihood is in parentheses. ${ }^{*} p<.01 .{ }^{* *} p<.001$. 


\section{Table 4 Hierarchical Regression Analysis for Moral Identity and Dispositional Doping Moral Disengagement} on Doping Likelihood in Study 2

\begin{tabular}{|c|c|c|c|c|c|c|}
\hline Step & Predictor variable & $B$ & SE B & $\boldsymbol{\beta}$ & $\Delta R^{2}$ & $\Delta F$ \\
\hline \multicolumn{7}{|c|}{$\begin{array}{l}\text { Doping likelihood under conditions } \\
\text { of low moral disengagement }\end{array}$} \\
\hline \multirow[t]{2}{*}{1} & Gender & -0.35 & 0.20 & -0.20 & .11 & $4.28 *$ \\
\hline & Social desirability & -0.08 & 0.04 & -0.26 & & \\
\hline \multirow[t]{2}{*}{2} & Moral identity & -0.21 & 0.10 & $-0.21 *$ & .27 & $14.32 * * *$ \\
\hline & Moral disengagement & 0.41 & 0.09 & $0.46 * * *$ & & \\
\hline 3 & Moral Identity $\times$ Moral Disengagement & -0.33 & 0.13 & $-0.23 *$ & .05 & $6.17 *$ \\
\hline \multicolumn{7}{|c|}{$\begin{array}{l}\text { Doping likelihood under conditions } \\
\text { of high moral disengagement }\end{array}$} \\
\hline \multirow[t]{2}{*}{1} & Gender & -0.33 & 0.34 & -0.11 & .10 & $3.85^{*}$ \\
\hline & Social desirability & -0.17 & 0.06 & $-0.29 *$ & & \\
\hline \multirow[t]{2}{*}{2} & Moral identity & -0.04 & 0.17 & -0.02 & .26 & $13.85^{* * *}$ \\
\hline & Moral disengagement & 0.80 & 0.15 & $0.52 * * *$ & & \\
\hline 3 & Moral Identity $\times$ Moral Disengagement & -0.25 & 0.24 & -0.10 & .01 & 1.06 \\
\hline
\end{tabular}

Note. The product terms were formed by multiplying mean-centered moral identity and mean-centered moral disengagement.

$* p<.05 . * * * p<.001$.

the doping moral disengagement-doping likelihood relationship under conditions where players had less opportunities to morally disengage (low moral disengagement condition), but not when opportunities to morally disengage were amplified (i.e., high moral disengagement condition). Altogether, these findings suggest that moral identity was negatively linked with athletes doping likelihood even if athletes are more inclined to morally disengage. That is, individuals who also reported that a moral self-schema was more central to (or an important part of) their identity, reported being less likely to use a banned substance even if they were more susceptible to justify doping. From a social cognitive perspective (e.g., Aquino et al., 2009), this is because such individuals would possess a higher activation potential of accessing their moral self-schema and its ability to influence moral behavior (e.g., reduce morally questionable behavior). However, this effect is only found under conditions of low moral disengagement (e.g., athletes with a supportive coach). When under conditions of higher moral disengagement (e.g., under coach pressure), the relationship between moral identity and doping likelihood was overridden. This is consistent with a social cognitive model perspective (e.g., Aquino \& Reed, 2002, Aquino et al., 2009), which suggests that when situations potentially decrease the accessibility of the moral self-schema within the working self-concept (i.e., in this case social interactions, which increase the potential and opportunity to morally disengage), this can result in a greater likelihood of committing transgressive behavior such as intentional doping. To extend the present findings, we aimed to test whether activating moral identity (via priming) influenced doping likelihood under conditions of low and high moral disengagement and whether these effects interact with dispositional moral identity and doping moral disengagement.

\section{Study 3}

\section{Methods}

Participants. Participants were 72 team sport players (53 men and 19 women), with an average age of $20.01(S D=3.80)$ years. The participants mainly competed in soccer $(n=26)$, rugby $(n=$ $14)$, field hockey $(n=6)$, tchoukball $(n=6)$, netball $(n=4)$, cricket $(n=4)$, basketball $(n=3)$, or lacrosse $(n=3)$. Participants competed in their respective sports at international/national $(17 \%)$, regional/county $(35 \%)$ and club (48\%) levels for an average of 8.30 $(S D=5.08)$ years.

Moral Identity and Moral Disengagement Manipulation. To activate moral identity, we employed an established priming method devised by Aquino et al. (2007, 2009), and previously applied in sport research (e.g., Kavussanu et al., 2015). Participants were presented with nine words and asked to think about each word and what it means to them. Next, participants copied each word by hand four times on a sheet of paper. Then, participants were asked to write a short story about themselves, which includes each of the nine words at least once and then were asked to read over their story and circle each of the nine words.

Participants in the moral identity group were asked to refer to moral traits, which were caring, compassionate, fair, friendly, generous, helpful, hardworking, honest, and kind. Reflecting and thinking about themselves with reference to these traits was expected to make the participants moral identity more salient in their working self-concept, thereby activating their moral identity (Aquino et al., 2009; Kavussanu et al., 2015). Participants in the control group were asked to refer to nine neutral words that were devoid of moral content, namely: book, car, chair, computer, desk, house, pen, street, and table. Considering and thinking about these words in relation to themselves was not expected to induce moral identity in the control group (Aquino et al., 2009; Kavussanu et al., 2015).

Following the moral identity prime, participants read each scenario to manipulate moral disengagement and completed the measures for moral judgment, anticipated guilt, and doping likelihood after each scenario, as per Study 2. Identical to Study 2, to control for order effects, we counterbalanced the moral disengagement conditions and the order of the scenarios.

Procedure. The procedure was identical to Study 2 whereby dispositional moral identity $(\alpha=.78)$, doping moral disengagement $(\alpha=.86)$, and social desirability were measured at least 3 days (but no more than 30 days) before the experimental session. However, the only difference in procedure was that participants were randomly allocated to the moral identity $(n=36 ; 28$ men and 8 

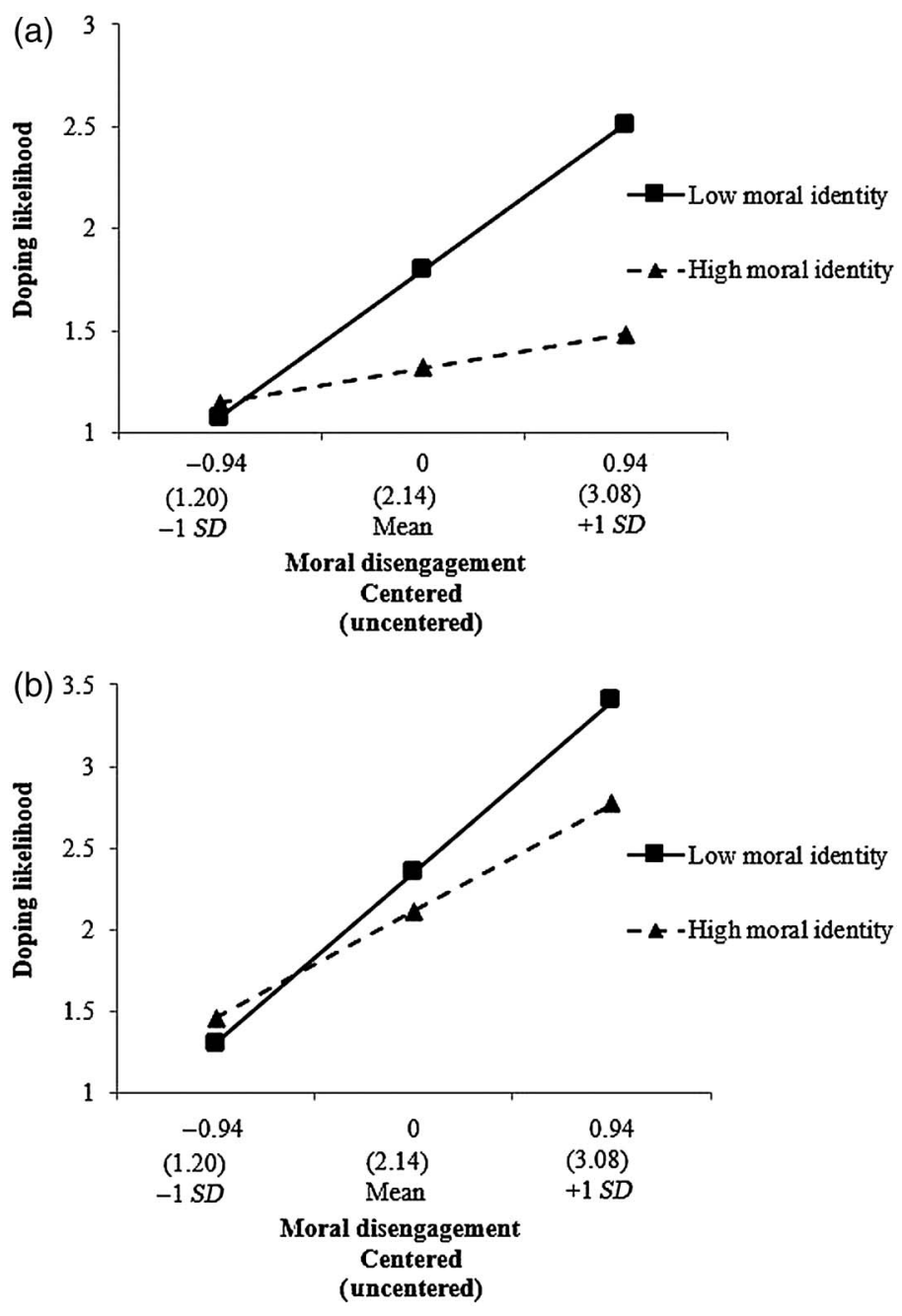

Figure 2 - The moderating effect of moral identity on the relationship between dispositional doping moral disengagement and doping likelihood under (a) the low moral disengagement and (b) the high moral disengagement condition in Study 2. The moderating effect of moral identity is only significant for the low moral disengagement condition.

women) or a control ( $n=36 ; 25$ men and 11 women) group and were administered the priming instructions depending on their assigned group (described above). ${ }^{5,6}$ Then, participants completed the scenarios to manipulate moral disengagement and completed the measures for anticipated guilt, moral judgment, and doping likelihood after each scenario. To help maintain the moral identity induction prior to each moral disengagement block, during the 3-min break between the low and high moral disengagement conditions participants were presented with the story they wrote for the moral identity (or control) induction and asked to read the story. After reading and completing the items for each of the six scenarios, participants were debriefed, thanked for their participation, and offered a $£ 5$ multistore voucher for taking part.

Manipulation Checks. The manipulation check for moral identity was similar to that used in previous research (e.g., Kavussanu et al., 2015) whereby participants were asked to reflect on their story and indicate on a 7-point scale anchored from 1 (to some extent) to 7 (to a great extent) how much the story reflected how they see themselves as a moral person, a student, and a member of an organization. A 2 Group (moral identity, control) $\times$ ANCOVA (controlling for gender and social desirability) revealed that the moral identity group $(M=6.03, S D=0.81)$ reported higher ratings for being a moral person than the control group $(M=3.33, S D=$ $1.79), F(1,68)=68.53, p<.001, \eta_{p}^{2}=.50$. There were no group differences for seeing the stories reflecting themselves as a student, or a person of an organization. During data inputting we also checked each participant's stories, and all participants adhered to the instructions for their respective experimental condition.

After each moral disengagement condition, participants completed the manipulation check items as per Study 2. A 2 (Moral disengagement condition) $\times 2$ (Moral identity group) mixed-design ANCOVA revealed participants reported higher moral disengagement if they were to take the substance in the situations for the high moral disengagement $(M=2.31, S D=1.38)$, compared with the low moral disengagement $(M=1.96, S D=1.04)$ condition, $F(1$, $68)=4.26 p=.04, \eta_{\mathrm{p}}^{2}=.06$. The effect size for this manipulation check was small to medium and weaker compared with Study 2, likely due to the focus and inclusion of the moral identity induction, which may have influenced the reported levels of moral disengagement. No significant interactions were found.

\section{Results}

Preliminary Analysis. There were only two cases of missing data $(0.03 \%)$, so we used the mean of the remaining items from the respective subscale when this occurred (Tabachnick \& Fidell, 2013). Screening for the normality of data via skewness and kurtosis values revealed no significant deviation from normality for any variable. However, doping likelihood in the low moral disengagement condition comprised of two extreme outliers, therefore, we winsorized these scores. No other variables comprised of extreme outliers.

Effect of Moral Identity and Moral Disengagement on Judgment, Guilt, and Doping Likelihood. To check for the effect of moral identity and moral disengagement on moral judgment, anticipated guilt, and doping likelihood, we conducted a mixed-design multivariate ANCOVA with moral identity as a between-subject factor, moral disengagement as a within-subject factor, and gender and social desirability as covariates. A significant multivariate effect for moral disengagement was found, $F(4,65)=7.08, p<.001, \eta_{p}^{2}=.30$. As presented in Table 5, follow-up repeated-measures ANCOVAs revealed significant differences for each variable. Specifically, similar to Study 2, participants perceived taking the banned substance as more acceptable and less morally wrong, anticipated feeling less guilt if they were to take a banned substance and reported greater likelihood to dope in the high moral disengagement condition compared with the low moral disengagement condition.

In terms of the moral identity prime, no significant main multivariate effect was found, $F(4,65)=0.84, p=.50, \eta_{p}^{2}=.05$, nor was there a significant Moral disengagement condition $\times$ Moral identity group interaction, $F(4,65)=1.13, p=.35, \eta_{\mathrm{p}}^{2}=.07$. Follow-up ANCOVAs confirmed no main effect for moral identity group for any variable.

Interactive Effects for Priming Moral Identity. Next, we examined the interactive effect of the Moral identity prime $\times$ Dispositional moral identity as well as Moral identity prime $\times$ Dispositional doping moral disengagement on doping likelihood across situations that differed in opportunities for moral disengagement. To address this aim, we used separate moderated hierarchical regression analyses for doping likelihood for each of the moral disengagement conditions. We included gender and social desirability as covariates in Step 1. Then, we included moral identity 
prime group, dispositional moral identity, and dispositional doping moral disengagement in Step 2. The product (interaction) terms for moral identity prime group $\times$ dispositional moral identity, Moral identity prime group $\times$ Doping moral disengagement, and Moral identity prime group $\times$ Dispositional moral identity $\times$ Doping moral disengagement were entered in Step 3. The results from these analyses are presented in Table 6.

For doping likelihood under conditions of low moral disengagement, the main effect for moral identity prime was noted, whereby the moral identity group reported lower doping likelihood than the control group. Doping moral disengagement was a positive predictor of doping likelihood, whereas dispositional moral identity was not a significant predictor of doping likelihood. In Step 3, a significant Moral identity prime group $\times$ Doping moral disengagement interaction was noted whereby the positive relationship between doping moral disengagement and doping likelihood was weaker in the high moral identity group, $b=0.20, \beta=0.49$, $t=3.35, p=.01$, compared with the low moral identity group, $b=$ $0.43, \beta=0.61, t=4.01, p<.001$. No Moral identity prime group $\times$ Dispositional moral identity interaction or Moral identity prime group $\times$ Dispositional moral identity $\times$ Doping moral disengagement was noted.

For doping likelihood under conditions of higher moral disengagement, the moral identity prime had no main effect on doping likelihood. Doping moral disengagement was a positive predictor, but dispositional moral identity was not a significant predictor of doping likelihood. No significant interaction effects were noted.

\section{Discussion}

Our findings from Study 3 indicate that activating moral identity reduced athletes' doping likelihood under conditions with low opportunities for moral disengagement, but not when athletes had

Table 5 Effects of MI and MD on Moral Judgment, Anticipated Guilt, and Doping Likelihood in Study 3

\begin{tabular}{|c|c|c|c|c|c|c|c|c|c|c|c|c|}
\hline & \multicolumn{4}{|c|}{ Low MD } & \multicolumn{4}{|c|}{ High MD } & \multicolumn{4}{|c|}{ Overall $(N=72)$} \\
\hline & \multicolumn{2}{|c|}{$\begin{array}{c}\text { Control } \\
\text { group } \\
(n=36)\end{array}$} & \multicolumn{2}{|c|}{$\begin{array}{c}\text { MI group } \\
(n=36)\end{array}$} & \multicolumn{2}{|c|}{$\begin{array}{l}\text { Control } \\
\text { group } \\
(n=36)\end{array}$} & \multicolumn{2}{|c|}{$\begin{array}{l}\text { MI group } \\
(n=36)\end{array}$} & \multirow[b]{2}{*}{ Low MD } & \multirow[b]{2}{*}{ High MD } & \multirow[b]{2}{*}{$F(1,69)$} & \multirow[b]{2}{*}{$\eta_{p}^{2}$} \\
\hline & $M$ & $S D$ & $M$ & $S D$ & $M$ & $S D$ & $M$ & $S D$ & & & & \\
\hline Morally wrong & 6.17 & 1.13 & 6.31 & 0.97 & 5.99 & 1.29 & 5.83 & 1.16 & $6.24(1.05)$ & $5.91(1.22)$ & $5.16^{*}$ & .07 \\
\hline Acceptable & 1.47 & 0.75 & 1.52 & 0.84 & 1.87 & 1.14 & 1.96 & 1.15 & $1.50(0.79)$ & $1.92(1.14)$ & $5.59 * * *$ & .08 \\
\hline Guilt & 4.01 & 0.97 & 4.13 & 0.87 & 3.80 & 1.11 & 3.77 & 0.93 & $4.07(0.92)$ & $3.79(1.02)$ & $8.32 * *$ & .11 \\
\hline Likelihood & 1.57 & 0.70 & 1.26 & 0.44 & 2.10 & 1.30 & 2.17 & 1.54 & $1.42(0.60)$ & $2.13(1.42)$ & $24.78 * * *$ & .27 \\
\hline
\end{tabular}

Note . $\mathrm{MI}=$ moral identity; $\mathrm{MD}=$ moral disengagement.

$* p<.05 . * * p<.01 . * * * p<.001$.

Table 6 Hierarchical Regression Analysis for MI and Doping MD on Doping Likelihood in Study 3

\begin{tabular}{|c|c|c|c|c|c|c|}
\hline Step & Predictor variable & $B$ & SE $B$ & $\boldsymbol{\beta}$ & $\Delta R^{2}$ & $\Delta \boldsymbol{F}$ \\
\hline \multicolumn{7}{|c|}{$\begin{array}{l}\text { Model 1: Doping likelihood } \\
\text { in low MD condition }\end{array}$} \\
\hline \multirow[t]{2}{*}{1} & Gender & -0.02 & 0.16 & -0.01 & .05 & 1.77 \\
\hline & Social desirability & -0.06 & 0.03 & -0.22 & & \\
\hline \multirow[t]{3}{*}{2} & MI group & -0.28 & 0.12 & $-0.24 *$ & .34 & $12.23 * * *$ \\
\hline & Dispositional MI & -0.02 & 0.10 & -0.02 & & \\
\hline & Doping MD & 0.31 & 0.06 & $0.53 * * *$ & & \\
\hline \multirow[t]{3}{*}{3} & MI Group $\times$ Dispositional MI & -0.17 & 0.22 & -0.14 & .04 & 1.49 \\
\hline & MI Group × Doping MD & -0.25 & 0.12 & $-0.31 *$ & & \\
\hline & MI Group $\times$ Dispositional MI × Doping MD & 0.16 & 0.18 & 0.09 & & \\
\hline \multicolumn{7}{|c|}{$\begin{array}{l}\text { Model 2: Doping likelihood } \\
\text { in high MD condition }\end{array}$} \\
\hline \multirow[t]{2}{*}{1} & Gender & -0.10 & 0.36 & -0.03 & .14 & $5.40^{* *}$ \\
\hline & Social desirability & -0.23 & 0.07 & $-0.36 * *$ & & \\
\hline \multirow[t]{3}{*}{2} & MI group & 0.11 & 0.28 & 0.04 & .25 & $8.73 * * *$ \\
\hline & Dispositional MI & -0.38 & 0.24 & -0.15 & & \\
\hline & Doping MD & 0.64 & 0.14 & $0.47 * * *$ & & \\
\hline \multirow[t]{3}{*}{3} & MI Group $\times$ Dispositional MI & -0.20 & 0.54 & -0.07 & .00 & 0.13 \\
\hline & MI Group × Doping MD & 0.07 & 0.29 & 0.04 & & \\
\hline & MI Group $\times$ Dispositional MI $\times$ Doping MD & -0.22 & 0.45 & -0.05 & & \\
\hline
\end{tabular}

Note. $\mathrm{MI}=$ moral identity; $\mathrm{MD}=$ moral disengagement.

$* p<.05 . * * p<.01 . * * * p<.001$. 
higher opportunities for moral disengagement. These findings replicate those noted in Study 2 for dispositional moral identity and doping likelihood. Moreover, similar to the interaction noted from Study 2, the positive relationship between doping moral disengagement and doping likelihood was attenuated for those whose moral identity was activated via priming in situations where players had low opportunities for moral disengagement. However, the interaction was not significant under situations when opportunities for moral disengagement were amplified. These findings suggest that activating one's moral identity may provide a benefit to reducing athletes' likelihood to dope under situations when opportunities for moral disengagement are low.

\section{General Discussion}

To address the profound lack of experimental-based research on antecedents of doping in sport (e.g., Ntoumanis et al., 2014), the aim of this research was to provide the first experimental evidence into the conjunctive effects of moral disengagement and moral identity on doping. Specifically, across three studies we tested the role of dispositional moral identity and doping moral disengagement on doping likelihood, moral judgment, and anticipated guilt. Moreover, we tested the effects of manipulating situational characteristics that trigger opportunities for moral disengagement and priming moral identity on doping likelihood.

\section{Moral Disengagement on Doping}

Overall, our findings indicate that a higher inclination for moral disengagement was associated with higher doping intention (Study 1) and likelihood (Studies 2 and 3); mainly reflective of medium to large effect sizes and similar to those noted in previous research (e.g., Boardley et al., 2017; Lucidi et al., 2008; Ring \& Kavussanu, 2018). Similarly, the effects of manipulating opportunities for situational moral disengagement on moral judgment, anticipated guilt, and doping likelihood were medium to large. Specifically, our research provides the first experimental evidence showing situational characteristics that amplify the potential for moral disengagement led athletes to judge doping as less wrong, more acceptable, and increased athletes' doping likelihood (Studies 2 and 3) partially via reducing anticipated guilt (Study 2). These findings are aligned to theoretical predictions that moral disengagement is a key factor in transgressive conduct, in part via reducing the regulatory role of anticipated guilt that typically refrain individuals from engaging in antisocial conduct (e.g., Bandura, 1991).

Our findings suggest that the manipulation of moral disengagement was effective. However, it should be acknowledged that the mean difference in the manipulation check scores for the moral disengagement manipulation was 0.38 in Study 2 (on a 1-7 scale), reflective of a medium to large effect. A weaker effect was noted in Study 3 which was likely due to the inclusion of the moral identity induction. These mean manipulation check scores for the high and low moral disengagement conditions were above and below the mean dispositional doping moral disengagement $(M=2.14, S D=0.95)$ scores, respectively. Moreover, moral disengagement scores tend to be relatively low (i.e., below the midpoint) as found in this research and previous studies (e.g., Kavussanu \& Ring, 2017; Lucidi et al., 2008). Therefore, on the whole, moral disengagement manipulation check scores could be argued to be within the expected range. Another reason for why the difference in manipulation check scores between conditions was not larger may be due to us aiming to change only one aspect for each scenario pertaining to each mechanism of moral disengagement. This was because (a) we wanted to manipulate only one relevant mechanism of moral disengagement for each scenario and (b) we did not want the differences in context to be too complex (or exaggerated) to help ensure situations were easy to understand and realistic to the sporting milieu. Given this study provides initial insight about the effects of manipulating moral disengagement, researchers may wish to extend our findings by manipulating multiple mechanisms of moral disengagement simultaneously within the same scenarios, which may accentuate the potential effectiveness of the moral disengagement manipulation.

Our findings are also aligned to previous research indicating that moral disengagement is a positive predictor of doping susceptibility (Hodge et al., 2013), doping intention (Lucidi et al., 2008), and doping likelihood (Ring \& Kavussanu, 2018). Our research extends previous work by testing moral disengagement as a process that can be influenced by situational characteristics. Specifically, although athletes with higher inclinations to morally disengage were associated with higher doping likelihood, if athletes are in situations where the potential for moral disengagement is amplified this also increased athletes' doping likelihood. This reinforces the importance of also considering the social environment (e.g., coaches, support personnel, parents) in anti-doping efforts (Backhouse, Griffiths, \& McKenna, 2018) rather than only targeting athlete's personal responsibility and individual agency in anti-doping programs. A multifaceted and comprehensive approach should take action on potential dopogenic environments (Backhouse et al., 2018) and reduce situations that may make athletes more susceptible to morally disengage, which in turn, could help reduce their likelihood of doping.

\section{Moral Identity and Doping}

Dispositional moral identity was negatively linked with doping likelihood in situations when the opportunities for moral disengagement were lower; even in individuals who have a greater inclination to morally disengage (Study 2). These findings are aligned to conceptual and theoretical arguments suggesting that moral identity provides a sense of moral motivation in the regulation of immoral conduct (e.g., Aquino et al., 2009), and the strength of associations was similar with those noted in previous research which found that the moral identity was negatively associated with doping likelihood (Kavussanu \& Ring, 2017). In addition, these findings support qualitative research that revealed a strong moral stance against cheating and valuing moral traits such as fairness, appeared to help reduce athletes' likelihood of doping (Erickson, McKenna, \& Backhouse, 2015). A key finding from our research pertains to the moderating role of dispositional moral identity (Study 2) as well as priming moral identity (Study 3) on the relationship between dispositional moral disengagement and doping likelihood. Our findings demonstrate similar effects to that found in the context of moral judgments toward severe retaliatory reactions to immoral acts or emotional reactions toward prisoner of war abuse (Aquino et al., 2007).

Interestingly, although dispositional moral identity and priming moral identity appeared to have some relationship or effect on athletes' doping likelihood, any effects or relationships were overridden in situations where opportunities for moral disengagement were amplified (Studies 2 and 3). These novel findings are aligned to the social cognitive model of moral identity (Aquino et al., 2009) and complement other recent research that revealed situational characteristics can influence the relationship between moral identity and doping (Ring et al., 2019). Therefore, our findings highlighted 
that in situations where potential for moral disengagement is amplified (Studies 2 and 3), any potential suppressing role of moral identity on doping was overridden. In view of this experimentally evidenced attenuation, the complexity of doping in sport is again highlighted and the importance of collective action to prevent anti-doping rule violations by all actors in the sporting system is reinforced. Doping prevention would benefit from responding to the interactions between athletes, their social networks, and the structures that directly influence how they operate and make decisions (e.g., Backhouse et al., 2018). For example, by addressing social and environmental conditions in a way that reduces the risk of coaches and their athletes advocating the use of prohibited substances (e.g., Whitaker, Backhouse, \& Long, 2017).

\section{Limitations and Future Research Directions}

Although this research provides a range of novel findings, they do need to be considered in light of some limitations. First, the research was reliant on self-report measures that can be prone to reporting bias, and we used a single item to measure doping intention in Study 1 which may not address the full extent of this construct. Also, given the experimental approach adopted in two studies, there is potential for demand characteristics. We attempted to counter this through measuring and controlling for social desirability, including filler items (e.g., anticipated pride items), measuring the extent doping behaviors were acceptable as well as wrong, and having at least 3 days between completion of the dispositional measures and the experimental session. Our findings could be extended further through longitudinal or intervention designs.

To measure dispositional doping moral disengagement, we used a scale developed by Lucidi et al. (2008). This was due to no other measures of doping moral disengagement being available at the point of project conception. However, although the scale comprised of an excellent model fit in Study 1, this scale has undergone limited psychometric testing (Lucidi et al., 2008). Therefore, researchers should consider employing more recently validated measures of doping moral disengagement that comprise more extensive psychometric support (e.g., Boardley et al., 2018; Kavussanu, Hatzigeorgiadis, Elbe, \& Ring, 2016) in future research.

In this research, we manipulated three mechanisms of moral disengagement which were predominantly reported in qualitative research to justify IPED use (e.g., Boardley \& Grix, 2014; Boardley et al., 2014). We decided to manipulate three mechanisms due to the within-subjects approach adopted to strengthen the experimental design, but we did not want to overwhelm participants by reading and rating responses in relation to too many situations. However, other mechanisms of moral disengagement have also been used to justify doping, namely euphemistic labeling, moral justification, and distortion of consequences. Moreover, the responses to each mechanism were in relation to one situation, and thereby participants were responding to specific contexts. Researchers could try and consider approaches to manipulate the other three mechanisms of moral disengagement used to justify doping and consider a wider range of situations to broaden the contexts that participants were asked to consider.

The manipulation check for moral disengagement was employed by adapting previously relevant items from the validated moral disengagement in sport scale (Boardley \& Kavussanu, 2007) to make them relevant to the context of doping. However, it should be acknowledged that we did not include items measuring the other mechanisms of moral disengagement. Therefore, it is possible that the scenarios may have potentially also manipulated the other mechanisms of moral disengagement that were not specifically targeted. In future, researchers may wish to consider employing a manipulation check comprising all mechanisms of moral disengagement to assess whether the scenarios may have also manipulated mechanisms not specifically targeted in the research.

The strength of the relationship between moral identity and doping likelihood found in this research, particularly under situations where there were greater opportunities to morally disengage, could also be moderated by self-regulatory efficacy (e.g., Bandura, 1991). Specifically, it is possible that athletes with high moral identity could still commit an anti-doping rule violation if they are not confident to resist temptations or social pressures to dope. Future research could consider the potential moderating role of self-regulatory efficacy and/or consider developing an experimental manipulation of self-regulatory efficacy to test its effect on athletes' doping likelihood. Given our findings, researchers could develop and test interventions that reduce the potential of athletes to morally disengage and increase moral identity; some suggestions that could be considered are provided below.

\section{Practical Implications}

Caution needs to be taken when extrapolating these findings to practical recommendations as this research was focused on doping intention/likelihood and behavior was not measured per se. However, some potential applied considerations can be offered. Our findings, in conjunction with previous research (e.g., Ring \& Kavussanu, 2018; Ring et al., 2019), suggest that enhancing athletes' moral identity and reducing the potential for moral disengagement could be a useful strategy within a comprehensive and multifaceted doping prevention framework. Recognizing that the role of moral identity on doping can be overridden under situations where athletes may be more susceptible to morally disengage, interventions would benefit from extending athlete-centered interventions with strategies targeted at support personnel (e.g., coaches) to reduce the likelihood of athletes' being exposed to situations where they are likely to morally disengage and engage in doping. In addition, one way that can help reduce dispositional moral disengagement is via enhancing personal responsibility and accountability for one's actions. To facilitate athletes' moral identity, approaches such as promoting a mental image about what it means to be a (moral) sportsperson could be accompanied by problemsolving activities (e.g., Hardy \& Carlo, 2011; Narvaez \& Lapsley, 2009) involving situations where players may be vulnerable to engage in doping (i.e., increased opportunities for moral disengagement). This reflexive process might assist athletes in preparing and taking personal responsibility to manage situations where the susceptibility for moral disengagement is heightened.

Anti-doping efforts would also benefit from workshops targeted at athlete support personnel and sport organizations facilitating awareness of "warning signs" and educating such stakeholders about how to avoid creating environments that heighten athletes' vulnerability to commit anti-doping rule violations. For instance, support personnel could be provided with scenarios that depict situations where opportunities for moral disengagement are amplified to stimulate action to reduce such vulnerabilities in their environment. However, future interventions would also need to consider the potential ambivalence of coaches (or support personnel) toward anti-doping education for such opportunities to be realized (e.g., Patterson, Backhouse, \& Lara-Bercial, 2019). 


\section{Conclusion}

This research provides the first step in acquiring the temporal sequencing of relationships between moral identity and moral disengagement on doping. Altogether, our findings highlight that moral disengagement is a moderate to strong positive predictor of doping likelihood both at a dispositional level and as a situational process and that moral identity may be negatively linked with doping in certain situations. However, the context-dependent nature of our findings adds further weight to calls to move beyond isolated athlete-centered interventions to also take action on the social environment. Research is now needed to test and refine approaches that can enhance moral identity, reduce athletes' dispositional propensity for moral disengagement as well as targeting key social agents (e.g., coaches) to reduce the potential of athletes (or as much as reasonably possible) being exposed to contexts where they are vulnerable to breaking the rules of sport by doping.

\section{Acknowledgments}

This research was carried out with funding support from the World AntiDoping Agency. The funder did not influence the design, conduct, analysis, or interpretation of the data in any way. Also, the authors would like to thank Luke Barnes and Alexandra J. Potts for their help with data collection, and Ian Boardley for his initial feedback on the scenario's used to manipulate moral disengagement.

\section{Notes}

1. Participants also completed measures for prosocial and antisocial behavior (Prosocial and Antisocial Behavior in Sport Scale; Kavussanu \& Boardley, 2009), moral disengagement (Moral Disengagement in Sport Scale_-short; Boardley \& Kavussnau, 2008), and a new doping measure that was being validated.

2. Although an a priori power calculation was not undertaken for Study 1 per se, related studies published at study conception linking doping moral disengagement with doping intention $(r=.45$; Lucidi et al., 2008), and moral identity with antisocial behavior in sport ( $r$ s $=-.33$ to -.49 ; Kavussanu et al., 2015; Sage et al., 2006) reflected at least medium effect sizes. Based on a medium effect size $(r=.30)$ with power $(1-\beta=0.80)$ and significance $(a=0.05)$ at conventional levels, a power calculation using $\mathrm{G}^{*}$ Power (Faul, Erdfelder, Lang, \& Buchner, 2007) estimated a sample size of 84 participants was required. More recent studies linking moral disengagement with doping susceptibility, likelihood, or reported behavior ( $r s=.33$ to .65 ; e.g., Boardley et al., 2017; Kavussanu \& Ring, 2017; Ring \& Kavussanu, 2018), and between moral identity and doping likelihood $(r=-.27$; Kavussanu \& Ring, 2017) have also found similar effect sizes. In addition, one previous study found a significant dispositional Moral identity $\times$ Advantageous comparison (mechanism of moral disengagement) interaction effect on moral judgment in a sample of 104 participants (Aquino et al., 2007; Study 1). Thus, based on the previous research, it was expected that the sample size in this study would have been sufficient to detect a significant correlation between moral identity and doping intention, and to examine a potential interaction effect.

3. An a priori power calculation to estimate the sample size for Study 2 was based on literature examining similar aims. In terms of testing for the effect of moral disengagement on doping likelihood, the only previous study that manipulated a mechanism of moral disengagement on antisocial behavior in sport (Stanger et al., 2013) revealed a significant medium to large effect $\left(\eta_{\mathrm{p}}^{2}=.19\right)$, and a medium effect for the mediating role of anticipated guilt $\left(K^{2}=.11\right.$; equivalent to $\left.\eta_{\mathrm{p}}^{2}=.11\right)$. Based on the lower effect size (i.e., $\eta_{p}^{2}=.11$ ), power calculation using $\mathrm{G}^{*}$ Power with conventional levels for power $(1-\beta=0.80)$ and significance $(a=0.05)$ yielded an estimated sample size of 68 for a within-subjects design. For examining the interactive effect, one previous study (Aquino et al., 2009; Study 2) found a significant interaction between dispositional moral identity and adapting situational factors (financial incentives) in a between-subjects design on intention to lie in a sample size of 55 participants (however, no specific effect size for the interaction was reported). Therefore, we aimed for a sample size divisible by 36 to allow for successful counterbalancing, and thereby we recruited 72 participants in this study.

4. Demographic information used to match responses included age, gender, main sport, competitive level, and last four digits of participants' mobile number to produce an anonymous code to match responses. Participants also completed a short picture viewing task at the start of the session before the manipulations to address a different study purpose. Specifically, participants were asked to rate their moral judgment and emotional involvement to pictures reflecting aggressive acts, pleasant stimuli (e.g., players celebrating), neutral pictures (e.g., players dribbling a ball), and athletes taking substances. Participants had a 5-min break after the picture viewing task before starting this experimental scenario task. It was not expected that this would have a confounding effect on the experimental manipulation during the same visit as participants were not asked questions specifically about moral disengagement, and the pictures reflected a range of different behaviors.

5. Identical to Study 2, participants completed a short picture viewing task at the start of the session before any manipulations (see Footnote 4). It was not expected that this would have a confounding effect on the experimental manipulation/inductions during the same visit as participants were not asked questions specifically examining either moral disengagement or moral identity during this picture viewing task, and the pictures reflected a range of different behaviors.

6. The estimated sample size for Study 3 was primarily based on two studies (Aquino et al., 2007, 2009). The first of which found a significant dispositional Moral identity $\times$ Moral identity prime interaction on intention to lie with a medium to large effect size $\left(\eta_{\mathrm{p}}^{2}=.19\right.$; Aquino et al., 2009). A power calculation using $G^{*}$ Power with conventional levels for power $(1-\beta=0.80)$ and significance $(a=0.05)$ revealed an estimated sample size of 32 participants. The second study is the only previous experimental study investigating the interactive effects of priming moral identity and dispositional advantageous comparison (mechanism of moral disengagement) albeit in relation to emotional reactions toward prisoner of war abuse (i.e., Aquino et al., 2007; Study 2, $N=69$ ), which found a significant interaction with an $R^{2}$ increase of .12 , over and above main effects for moral identity and advantageous comparison. A power calculation using conventional levels and the effect size noted for the interaction effect $\left(R^{2}\right.$ increase $\left.=.12\right)$ estimated we required 74 participants. To ensure successful counterbalancing of the manipulation across both low and high moral identity groups (i.e., divisible by 36 ) we recruited a sample size of 72 participants, which was similar to the estimated sample to detect a significant interaction effect.

\section{References}

Aiken, L.S., \& West, S.G. (1991). Multiple regression: Testing and interpreting interactions. Newbury Park, UK: Sage.

Aquino, K., Freeman, D., Reed, A., Lim, V.K.G., \& Felps, W. (2009). Testing a social-cognitive model of moral behavior: The interactive influence of situations and moral identity centrality. Journal of 
Personality \& Social Psychology, 97, 123-141. PubMed ID: 19586244 doi:10.1037/a0015406

Aquino, K., \& Reed, A. (2002). The self-importance of moral identity. Journal of Personality \& Social Psychology, 83, 1423-1440. PubMed ID: 12500822 doi:10.1037/0022-3514.83.6.1423

Aquino, K., Reed, A., Thau, S., \& Freeman, D. (2007). A grotesque and dark beauty: How moral identity and mechanisms of moral disengagement influence cognitive and emotional reactions to war. Journal of Experimental Social Psychology, 43, 385-392. doi:10. 1016/j.jesp.2006.05.013

Backhouse, S.H., Griffiths, C., McKenna, J. (2018). Tackling doping in sport: a call to take action on the dopogenic environment. British Journal of Sports Medicine, 52, 1485-1486. PubMed ID: 28666982 doi:10.1136/bjsports-2016-097169

Bandura, A. (1991). Social cognitive theory of moral thought and action. In W.M. Kurtines\& J.L. Gewirtz (Eds.), Handbook of moral behavior and development (pp. 45-103). Hillsdale, NJ: Erlbaum.

Barkoukis, V., Lazuras, L., Lucidi, F., \& Tsorbatzoudis, H. (2015). Nutrition supplement and doping use in sport: Possible underlying social cognitive processes. Scandinavian Journal of Medicine \& Science in Sports, 25, e582-e588. PubMed ID: 25556707 doi:10. 1111/sms. 12377

Barkoukis, V., Lazuras, L., Tsorbatzoudis, H., \& Rodafinos, A. (2013). Motivational and social cognitive predictors of doping intentions in elite sport: An integrated approach. Scandinavian Journal of Medicine \& Science in Sports, 23, e330-e340. PubMed ID: 23574429 doi:10.1111/sms.12068

Boardley, I.D., \& Grix, J. (2014). Doping in bodybuilders: A qualitative investigation of facilitative psychosocial processes. Qualitative Research in Sport, Exercise, \& Health, 6, 422-439. doi:10.1080/ 2159676X.2013.766809

Boardley, I.D., Grix, J., \& Dewar, A.J. (2014). Moral disengagement and associated processes in performance-enhancing drug use: A national qualitative investigation. Journal of Sports Sciences, 32, 836-844. PubMed ID: 24405120 doi:10.1080/02640414.2013.862842

Boardley, I.D., \& Kavussanu, M. (2007). Development and validation of the moral disengagement in sport scale. Journal of Sport \& Exercise Psychology, 29, 608-628. PubMed ID: 18089895 doi:10.1123/jsep. 29.5.608

Boardley, I.D., \& Kavussanu, M. (2008). The moral disengagement in sport scale-Short. Journal of Sports Sciences, 26, 1507-1517. PubMed ID: 18972249 doi:10.1080/02640410802315054

Boardley, I.D., \& Kavussanu, M. (2011). Moral disengagement in sport. International Review of Sport \& Exercise Psychology, 4, 93-108. doi:10.1080/1750984X.2011.570361

Boardley, I.D., Smith, A.L., Mills, J.P., Grix, J., \& Wynne, C. (2017). Empathic and self-regulatory processes governing doping behavior. Frontiers in Psychology, 8, 1495. PubMed ID: 29018370 doi:10. 3389/fpsyg.2017.01495

Boardley, I.D., Smith, A.L., Mills, J.P., Grix, J., Wynne, C., \& Wilkins, L. (2018). Development of moral disengagement and self-regulatory efficacy assessment relevant to doping in sport and exercise. Psychology of Sport \& Exercise, 36, 57-70. doi:10.1016/j.psychsport. 2018.01.007

Bredemeier, B.J., \& Shields, D.L. (1986). Athletic aggression: An issue of contextual morality. Sociology of Sport Journal, 3, 15-28. doi:10. 1123/ssj.3.1.15

Erickson, K., McKenna, J., \& Backhouse, S.H. (2015). A qualitative analysis of the factors that protect athletes against doping in sport. Psychology of Sport \& Exercise, 16, 149-155. doi:10.1016/j. psychsport.2014.03.007
Faul, F., Erdfelder, E., Lang, A.-G., \& Buchner, A. (2007). G*Power 3: A flexible statistical power analysis program for the social, behavioral, and biomedical sciences. Behavior Research Methods, 39, 175-191. PubMed ID: 17695343 doi:10.3758/BF03193146

Hardy, S.A., \& Carlo, G. (2011). Moral identity: What is it, how does it develop, and is it linked to moral action? Child Development Perspectives, 5, 212-218. doi:10.1111/j.1750-8606.2011.00189.x

Hayes, A.F. (2009). Beyond Baron \& Kenny: Statistical mediation analysis in the new millennium. Communication Monographs, 76, 408420. doi:10.1080/03637750903310360

Hodge, K., Hargreaves, E.A., Gerrard, D., \& Lonsdale, C. (2013). Psychological mechanisms underlying doping attitudes in sport: Motivation and moral disengagement. Journal of Sport \& Exercise Psychology, 35, 419-432. PubMed ID: 23966451 doi:10.1123/jsep. 35.4.419

Huybers, T., \& Mazanov, J. (2012). What would Kim do: A choice study of projected athlete doping considerations. Journal of Sport Management, 26, 322-334. doi:10.1123/jsm.26.4.322

Kavussanu, M., \& Boardley, I.D. (2009). The prosocial and antisocial behavior in sport scale. Journal of Sport \& Exercise Psychology, 31, 97-117. PubMed ID: 19325190 doi:10.1123/jsep.31.1.97

Kavussanu, M., Boardley, I.D., Sagar, S., \& Ring, C. (2013). Bracketed morality revisited: How do athletes behave in two contexts? Journal of Sport \& Exercise Psychology, 35, 449-463. PubMed ID: 24197713 doi:10.1123/jsep.35.5.449

Kavussanu, M., Hatzigeorgiadis, A., Elbe, A-M., \& Ring, C. (2016). The moral disengagement in doping scale. Psychology of Sport \& Exercise, 24, 188-198. doi:10.1016/j.psychsport.2016.02.003

Kavussanu, M., \& Ring, C. (2017). Moral identity predicts doping likelihood via moral disengagement and anticipated guilt. Journal of Sport \& Exercise Psychology, 39, 293-301. PubMed ID: 29111866 doi:10.1123/jsep.2016-0333

Kavussanu, M., Stanger, N., \& Ring, C. (2015). The effects of moral identity on moral emotion and antisocial behavior in sport. Sport, Exercise, \& Performance Psychology, 4, 268-279. doi:10.1037/spy0000040

Kaye, M.P., \& Boardley, I.D. (2012). Beyond punishment: Doping, deterrence, and moral disengagement. Journal of Sports Medicine \& Doping Studies, 2, e127. doi:10.4172/2161-0673.1000e127

Kirby, K., Moran, A., \& Guerin, S. (2011). A qualitative analysis of the experiences of elite athletes who have admitted to doping for performance enhancement. International Journal of Sports Policy, 3, 205-224. doi:10.1080/19406940.2011.577081

Koller, D.L. (2008). From medals to morality: sportive nationalism and the problem of doping in sports. Marquette Sports Law Practice, 19, 93-124.

Lucidi, F., Zelli, A., Mallia, L., Grano, C., Russo, P.M., \& Violani, C. (2008). The social-cognitive mechanism regulating adolescents' use of doping substances. Journal of Sports Sciences, 26, 447-456. PubMed ID: 18274942 doi:10.1080/02640410701579370

Marschall, D.E., Saftner, J., \& Tangney, J.P. (1994). The state shame and guilt scale. Fairfax, VA: George Mason University.

Montoya, A.K., \& Hayes, A.F. (2017). Two condition within-participant statistical mediation analysis: A path-analytic framework. Psychological Methods, 22, 6-27. PubMed ID: 27362267 doi:10.1037/ met0000086

Moore, C. (2015). Moral disengagement. Current Opinion in Psychology, 6, 199-204. doi:10.1016/j.copsyc.2015.07.018

Narvaez, D., \& Lapsley, D.K. (2009). Moral identity, moral functioning, and the development of moral character. In D.M. Bartels, C.W. Bauman, L.J. Skitka, \& D.L. Medin (Eds.), The psychology of learning and motivation (pp. 237-274). Burlington, VT: Academic Press. 
Ntoumanis, N., Ng, J.Y., Barkoukis, V., \& Backhouse, S. (2014). Personal and psychosocial predictors of doping use in physical activity settings: A meta-analysis. Sports Medicine, 44, 1603-1624. PubMed ID: 25138312 doi:10.1007/s40279-014-0240-4

Patterson, L.B., Backhouse, S.H., \& Lara-Bercial, S. (2019). Examining coaches' experiences and opinions of anti-doping education. International Sport Coaching Journal, 6, 145-159. doi:10.1123/iscj.20180008

Pope, H.G., Wood, R.I., Rogol, A., Nyberg, F., Bowers, L., \& Bhasin, S. (2013). Adverse health consequences of performance-enhancing drugs: An Endocrine Society scientific statement. Endocrine Reviews, 35, 341-375. doi:10.1210/er.20131058

Reynolds, W.M. (1982). Development of reliable and valid short forms of the Marlowe-Crowne social desirability scale. Journal of Clinical Psychology, 38, 119-125. doi:10.1002/1097-4679(198201)38:1\% 3C119::AID-JCLP2270380118\%3E3.0.CO;2-I

Ring, C., \& Kavussanu, M. (2018). The role of self-regulatory efficacy, moral disengagement and guilt on doping likelihood: A social cognitive theory perspective. Journal of Sports Sciences, 36, 578584. PubMed ID: 28481691 doi:10.1080/02640414.2017.1324206

Ring, C., Kavussanu, M., Lucidi, S., \& Hurst, P. (2019). Effects of personal and situational factors on self-referenced doping likelihood. Psychology of Sport \& Exercise, 41, 29-35. doi:10.1016/j. psychsport.2018.11.003

Sage, L., Kavussanu, M., \& Duda, J. (2006). Goal orientations and moral identity as predictors of prosocial and antisocial functioning in male association football players. Journal of Sports Sciences, 24, 455-466. PubMed ID: 16608760 doi:10.1080/02640410500244531

Stanger, N., Kavussanu, M., Boardley, I.D., \& Ring, C. (2013). The influence of moral disengagement and negative emotion in the regulation of antisocial behavior. Sport, Exercise and Performance Psychology, 2, 117-129. doi:10.1037/a0030585

Stets, J.E., \& Carter, M.J. (2011). The moral self: Applying identity theory. Social Psychology Quarterly, 74, 192-215. doi:10.1177/01902 72511407621

Tabachnick, B.G., \& Fidell, L.S. (2013). Using multivariate statistics (6th ed.). Boston, MA: Pearson Education.

West, S.G., Finch, J.F., \& Curran, P.J. (1995). Structural equation models with non normal variables: Problem and remedies. In R.H. Hoyle (Ed.), Structural equation modeling: Concepts, issues, and applications (pp. 56-75). Newbury Park, CA: Sage.

Whitaker, L., Backhouse, S.H., \& Long, J. (2017). Doping vulnerabilities, rationalisations and contestations: The lived experience of national level athletes. Performance Enhancement and Health, 5, 134-141. doi:10.1016/j.peh.2017.06.001

Whitaker, L., Long, J., Petróczi, A., \& Backhouse, S. (2013). Using prototype willingness model to predict doping in sport. Scandinavian Journal of Medicine \& Science in Sports, 24, e398-e405. doi:10. 1111/sms. 12148

World Anti-Doping Agency. (2015). World anti-doping code. Retrieved from https://www.wa-da-ama.org/sites/default/files/resources/files/ wada-2015-world-anti-doping-code.pdf 


\section{Appendix: Moral Disengagement Manipulation Scenarios}

\section{Low Moral Disengagement Condition}

Displacement of responsibility: Imagine that recently your sport performances have not been as good as last season. Your coach has been very supportive and loyal, instilling you with confidence and encouragement that you will soon rediscover your form. You have noticed that one of your teammates' performances have really improved recently. After practice one day, your teammate tells you that he/she has been using a banned performance-enhancing substance, and there are no tests to detect it.

Diffusion of responsibility: A teammate introduces you to a substance that and you are aware that its use can greatly help improve sports performance and there are no tests to detect it. You are aware that use of this substance is banned in your sport, but you do not suspect that any of your teammates or opponents is using such substances to give them an advantage.

Advantageous comparison: You have a very important match approaching and have become aware of a banned substance that helps to improve performance and there are no tests that can detect it. You perceive that this substance would only provide a slight advantage to your performance, and you do not suspect any of your teammates or opponents deliberately commit rule-breaking behaviors to gain an advantage.

\section{High Moral Disengagement Condition}

Displacement of responsibility: Imagine that recently your sport performances have not been as good as last season and particularly not to the level that your coach expects. Your coach is putting intense pressure on you to perform better and threatens that he/she may drop you from the squad if you do not find a way to improve your performances soon. You have noticed that one of your teammates' performances have really improved recently. After practice one day, your teammate tells you that he/she has been using a banned performance-enhancing substance and there are no tests to detect it.

Diffusion of responsibility: A teammate introduces you to a substance, and you are aware that its use can greatly help improve sports performance and there are no tests to detect it. You are aware that use of this substance is banned in your sport, but you suspect that many of your teammates and opponents that you compete against are using such substances to give them an advantage.

Advantageous comparison: You have a very important match approaching and have become aware of a banned substance that helps to improve performance and there are no tests that can detect it. You perceive that this substance would only provide a slight advantage to your performance, particularly in comparison with some of your teammates and opponents whom you suspect trying to deliberately injure other players to gain an advantage.

Note. The texts in italics are the sections that differed either to increase moral disengagement (in high moral disengagement conditions) or reduce moral disengagement (low moral disengagement conditions). 